\title{
The Effect of Solvent and Counterion Variation on Inverse Micelle CMCs in Hydrocarbon Solvents
}

\author{
Gregory N. Smith ${ }^{\mathrm{a}, 1}$, Paul Brown ${ }^{\mathrm{a}, 2}$, Craig James ${ }^{\mathrm{a}, 3}$, Sarah E. Rogers ${ }^{\mathrm{b}}$, Julian Eastoe ${ }^{\mathrm{a}}$ \\ ${ }^{a}$ School of Chemistry, University of Bristol, Cantock's Close, Bristol, BS8 1TS, United Kingdom \\ ${ }^{b}$ ISIS-STFC, Rutherford Appleton Laboratory, Chilton, Oxon, OX11 OQX, United Kingdom
}

\begin{abstract}
Critical micelle concentrations (CMCs) for the formation of inverse micelles have been determined for anionic surfactants in nonpolar, hydrocarbon solvents. Sodium dioctylsulfosuccinate (Aerosol OT or AOT) was chosen as the model surfactant, with systematic variations in both the solvent (benzene, cyclohexane, and dodecane) and the surfactant counterion (sodium and tetrapropylammonium). Recent work (Langmuir 2013 29 3352-3258) has shown that high-resolution small-angle neutron scattering (SANS) measurements can be used to directly determine the presence or absence of aggregates in solution. No variation in the value of the $\mathrm{CMC}$ was found within the resolution of the measurements for changing either solvent or counterion; some effects on the structure of inverse micelles were observed. This lack of a significant difference in the onset of inverse micellization with changes to the molecular species is surprising, and the implications on the solvophobic effect in nonpolar solvents are discussed.
\end{abstract}

Keywords: Nonpolar solvents; Inverse micelles; Small-angle neutron scattering

\footnotetext{
Email address: julian.eastoe@bristol.ac.uk (Julian Eastoe)

${ }^{1}$ Current address: Department of Chemistry, University of Sheffield, Brook Hill, Sheffield, South Yorkshire, S3 7HF, United Kingdom

${ }^{2}$ Current address: Department of Chemical Engineering, Massachusetts Institute of Technology, Cambridge, Massachusetts 02139, United States of America

${ }^{3}$ Current address: Department of Chemistry and CSGI, University of Florence, 50019 Sesto Fiorentino, Firenze, Italy
} 


\section{Graphical abstract}
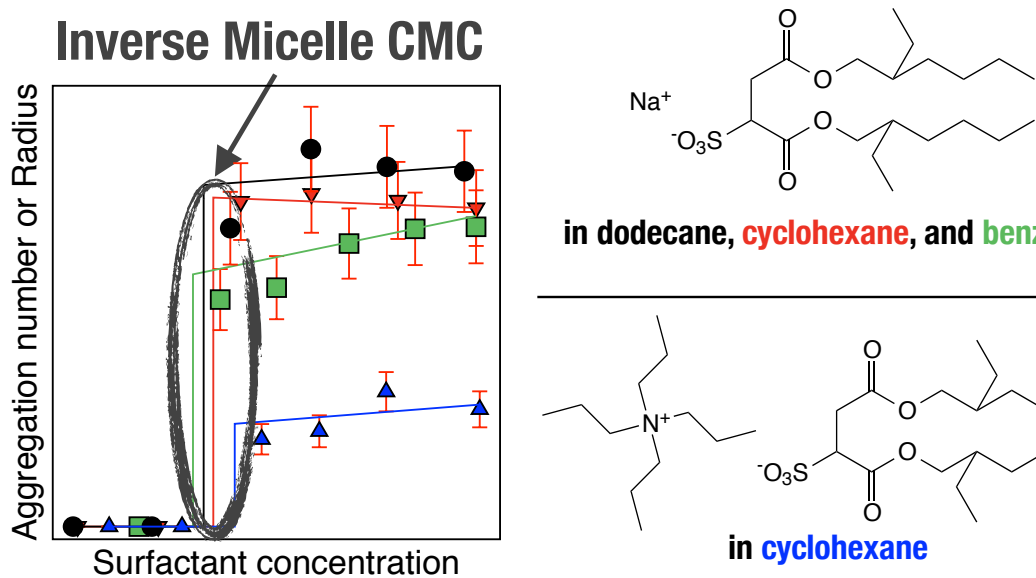

in dodecane, cyclohexane, and benzene

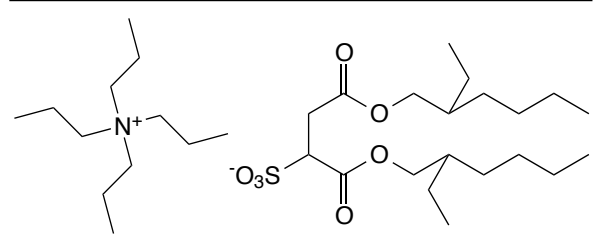

in cyclohexane

\section{Introduction}

Klevens proposed a now well-known relationship between the critical micelle concentration (CMC) of a homologous series of linear chain surfactants in water and the number of carbon atoms in the tails, $n_{c}[1,2$.

$$
\log (\mathrm{CMC})=A_{K}-B_{K} n_{c}
$$

$A_{K}$ depends on the surfactant headgroup (the "hydrophilic" part of the molecule). For ionic hydrocarbons, the value of $B_{K}$ is empirically $\log 2$, and it scales the effect of the length of the hydrocarbon chain (the "hydrophobic" part) on the value of the CMC. Literature data for CMCs of sodium dialkylsulfosuccinate and alkylsulfate salts with different $n_{c}$ values in water are shown in Figure 1. $\log (\mathrm{CMC})$ is indeed directly proportional to $n_{c}$, and the gradient is -0.31 for dialkylsulfosuccinates and is -0.29 for alkylsulfates ( $\log 2 \approx$ 0.30). Increasing the surfactant chain length decreases the CMC. Tanford has shown that micellization in water occurs due to the hydrophobic effect, and the contribution of adding hydrocarbon groups to the free energy of micellization can be calculated from $R T\left(\mathrm{~d} \ln \mathrm{CMC} / \mathrm{d} n_{c}\right)$. The decrement in Gibbs energy of micellization is $\sim 3 \mathrm{~kJ} \mathrm{~mol}^{-1}$ per $\mathrm{CH}_{2}$ group, although it is reduced for ionic surfactants in water due to the background ionic strength of the free surfactant 3,4 .

Changing from water to a nonpolar solvent (relative permittivity, $\epsilon_{r} \approx 2$ ) significantly influences the nature of the surfactant-solvent interactions; the terms hydrophilic and hydrophobic lose meaning. Rather, the two parts of the molecule are better described as "solvophilic" and "solvophobic." In nonpolar solvents, the alkyl tails are solvophilic (in water, hydrophobic), and the ionic headgroups are solvophobic (in water, hydrophilic). This has the consequence of inverting the aggregate structure; sodium dioctylsulfosuccinate (Aerosol OT or AOT), for example, forms inverse micelles and reverse water-in-oil (w/o) microemulsions 8. The Klevens equation can still apply in nonpolar solvents, as long as $n_{c}$ is replaced by the number of 


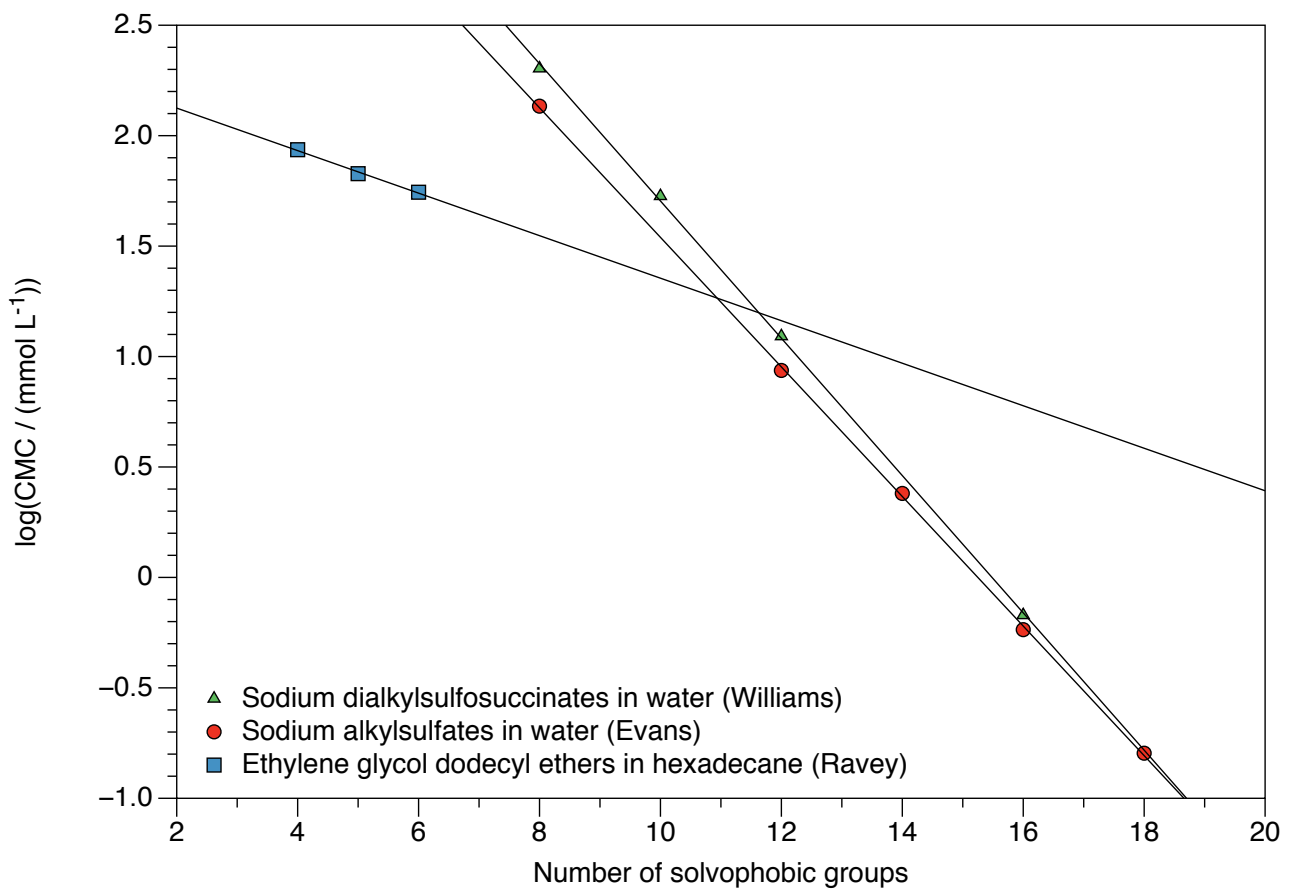

Figure 1: Klevens equation plots (log $(\mathrm{CMC})$ versus number of solvophobic groups) for three systems of amphiphiles (sodium dialkylsulfosuccinates in water [5], sodium alkylsulfates in water [6], and ethylene glycol dodecyl ethers in hexadecane [7]). For ionic surfactants in water, the trend is known from Klevens's publications [1, 2], and it has a gradient of $\sim \log 2$. For the nonionic surfactants in hexadecane, the CMC also depends on the number of solvophobic groups, although the gradient is shallower. 
solvophobic groups. Figure 1 shows how the CMC varies with the number of ethylene oxide solvophobic groups for nonionic surfactants in hexadecane [7. There is still a linear relationship between $\log (\mathrm{CMC})$ and the number of solvophobic groups, but the slope is now different. The nonaqueous solvent $B_{K}$ per solvophobic group for ethylene glycol dodecyl ethers in hexadecane is 0.10 or $\log 1.2$. Solvophobicity is an additive property in alkane solvents, just like in water, and it is directly related to the surfactant molecular structure. The decrement in Gibbs energy of micellization or inverse micellization per solvophobic group is less for ethylene glycol dodecyl ether surfactants in hexadecane than for sodium alkylsulfate surfactants in water. This group contribution is found to be $\sim 0.5 \mathrm{~kJ} \mathrm{~mol}^{-1}$ per ethylene oxide group in hexadecane, compared to $\sim 3 \mathrm{~kJ} \mathrm{~mol}^{-1}$ for $\mathrm{CH}_{2}$ groups in water, despite the larger number of atoms per solvophobic group.

In this paper, derivatives of the model anionic surfactant AOT are studied in nonpolar solvents. This surfactant does not have a solvophobic increment like the surfactants shown in Figure 1, but AOT is an interesting and well-studied model surfactant that is important in numerous applications as, for example, a microemulsion stabilizer [8], as a micellar nanoreactor [9], or as a charge control agent [10]. Understanding how this surfactant forms inverse micelles at low concentrations is important for efficient and controllable use in applications. Given that there is no solvophobic group to vary in AOT, how would the Klevens equation apply to such a system? The solvophilicity index $\left(A_{K}\right)$ now relates to the hydrocarbon tails, and the solvophobicity parameter $\left(B_{K}\right)$ now relates to the surfactant headgroups. As solvophobicity is driven by the ionic group, $B_{K} n_{c}$ now needs to be thought of as a single variable. The equation now takes the form $\log (\mathrm{CMC})=A_{K}-B_{K}$. This two-parameter equation demonstrates the concept that aggregation is a balance between solvophilicity $\left(A_{K}\right)$ and solvophobicity $\left(B_{K}\right)$. The solvophobicity of the AOT cannot be readily changed as it is defined by the molecular structure; on the other hand, the solvophilicity of AOT can be readily changed by varying the solvent quality [11].

The existence of a $\mathrm{CMC}$ for inverse micelle formation is still debated [12, 13]. (In this paper, the CMC from now on refers to the critical concentration for inverse micelle formation, unless otherwise stated.) Experimentalists, however, have succeeded in measuring transitions that are consistent with a step change from monomers to inverse micelles at a critical concentration [14 22]. Accurate and consistent values of CMCs for inverse micelle formation are lacking in the literature. Several groups have used techniques as disparate as iodine solubilization and mercury interfacial tension to measure CMCs for AOT in different organic solvents, but the results are highly variable between the different studies [15, 16, 19, 20, 23, 24]. Measuring CMCs in organic solvents is challenging experimentally. Techniques frequently used to detect CMCs in water (conductivity, surface tension, or dye solubilization) have drawbacks that make them illsuited to detect inverse micelle CMCs in organic solvents [22. Due to high contrast and sensitivity, smallangle scattering measurements provide perhaps the strongest experimental evidence for a critical micelle concentration in aliphatic and aromatic nonpolar solvents [18, 22, 25, 26]. 
To compare aggregation of surfactants in nonpolar solvents to water, suitable molecular variations need to be studied, analogous to the $B_{K} n_{c}$ term in Equation 1 . For surfactants in water, extending the tail length makes the surfactant more solvophobic. However, changing the surfactant tails is not viable in nonpolar solvents; they are now the solvophilic groups. Instead, changes to the counterion and the solvent are made. The CMC of AOT in three organic solvents (benzene- $d_{6}$, cyclohexane- $d_{12}$, and dodecane- $d_{26}$ ) and of an analogue, where the $\mathrm{Na}^{+}$counterions have been exchanged for tetrapropylammonium (TPA-AOT) cations in cyclohexane- $d_{12}$, were measured. Varying the identity of the solvent would be expected to modify the solvent quality for surfactant tails, and changing from a hard counterion $\left(\mathrm{Na}^{+}\right)$to a soft counterion $\left(\mathrm{TPA}^{+}\right)$ would be expected to modify the surfactant solvophobicity. The effect of varying surfactant structure on the formation of micelles in water is well-known, but the effect of these variations on the formation of inverse micelles in nonpolar solvents cannot be predicted. High-resolution SANS measurements have been used to measure the CMCs for inverse micellar systems, as recently done for a nonionic surfactant $\left(\mathrm{C}_{12} \mathrm{E}_{5}\right)$ and sodium AOT in cyclohexane- $d_{12}[22$. Values of the CMC measured by this method are among the lowest in the literature, and this shows the importance of using high-resolution SANS measurements. The results help to develop new understanding of the nature of aggregation in nonpolar solvents.

\section{Methods}

\subsection{Sample Preparation}

Sodium dioctylsulfosuccinate (AOT, 98\%, Aldrich) was purified in dried diethyl ether and dried in vacuum at $70^{\circ} \mathrm{C}$ for 12 hours before use. Tetrapropylammonium AOT (TPA-AOT) was prepared as previously described [27]. Deuterium-labeled TPA-AOT- $d_{34}$ was prepared in the same way, but 2-ethylhexanol- $d_{17}$ (provided by the Oxford Isotope Facility) was used to synthesize AOT- $d_{34}$ [28]. Benzene- $d_{6}(99.6$ atom \% D, Aldrich), cyclohexane- $d_{12}$ (> 99.50 atom \% D, Apollo Scientific), and dodecane- $d_{26}$ (98\%, Cambridge Isotope Laboratories) were used as supplied.

Stock solutions of surfactant in nonpolar solvent were made using $5 \mathrm{~mL}$ volumetric flasks with a concentration of $\sim 10 \mathrm{mmol} \mathrm{L}^{-1}$. Stock solutions were then diluted volumetrically to give the desired final concentrations. (The actual concentrations of stock and diluted solutions are given in the Supplementary Data file.) Solutions of NaAOT in cyclohexane and dodecane as well as TPA-AOT in cyclohexane were diluted with a logarithmic step of 4 , and solutions of NaAOT in benzene were diluted with a logarithmic step of 3 .

\subsection{Small-Angle Neutron Scattering (SANS)}

SANS measurements were carried out on the Sans2d small-angle diffractometer at the ISIS Pulsed Neutron Source (STFC Rutherford Appleton Laboratory, Didcot, U.K.) [29, 30]. A simultaneous $Q$-range of 0.004-0.80 $\AA^{-1}$ was achieved by utilizing an incident wavelength range of $1.75-16.5 \AA$ and employing an 
instrument setup of $\mathrm{L} 1=\mathrm{L} 2=4 \mathrm{~m}$, with the $1 \mathrm{~m}^{2}$ detector offset vertically $150 \mathrm{~mm}$ and sideways $269 \mathrm{~mm}$. $Q$ is the momentum transfer vector, defined in Equation 2 below, where $\theta$ is half the scattering angle and $\lambda$ is the incident neutron wavelength.

$$
Q=\frac{4 \pi \sin \theta}{\lambda}
$$

SANS experimental and data reduction procedures are described elsewhere [22]. For reasons of experimental setup, SANS of TPA-AOT- $d_{34}$ was measured with an $8 \mathrm{~mm}$ diameter beam rather than the $12 \mathrm{~mm}$ beam that was used for all other samples.

The intensity $I(Q)$ is a function of the aggregate volume fraction $(\phi)$, the volume of the aggregate $\left(V_{p}\right)$, the scattering-length density contrast $(\Delta \rho)$, the scattering form factor $(P(Q))$, and the scattering structure factor $(S(Q))$ 31.

$$
I(Q)=\phi V_{p} \Delta \rho^{2} P(Q) S(Q)
$$

No $S(Q)$ contribution was required to fit the data, indicating that the aggregates do not significantly interact in these low dielectric, nonpolar solvents. Data have been fit to either a sphere [32] or a core-shell sphere 32 34] form factor using the SasView small-angle scattering analysis software package [35. The scale factor (equal to $\phi$ ), the aggregate radius $(r)$, and the flat background were allowed to vary until the best fit was achieved. The fit was considered "good" as scale factors were approximately $70 \%$ of the experimental volume fraction. The fit values for radii are considered to have a certainty of $\pm 1 \AA$. All form factors included size dispersity using a Schulz distribution [36, 37.

\subsection{Molecular volume}

Computational chemistry calculations were performed using Gaussian 09 38. Geometry optimization was performed using DFT at the B3LYP/6-31+G(d) level of theory along with molecular volumes using tight criteria. The molecular volumes were calculated to be $512 \AA^{3}$ for the AOT anion and $281 \AA^{3}$ for the TPA cation. The molecular volume of the sodium cation was $44 \AA^{3}$, taken from DFT/B3LYP calculations in the literature [39. This gives molecular volumes for the whole surfactant molecules of $556 \AA^{3}$ for NaAOT and $793 \AA^{3}$ for TPA-AOT; the molecular volume of NaAOT compares favorably with the value calculated from the solid state properties $\left(671 \AA^{3}\right)$ [1].

\section{Results and Discussion}

Two molecular variations that influence the solvophobic interaction between surfactant and solvent have been studied. The effect of varying the solvent (cyclohexane- $d_{12}$, benzene- $d_{6}$, or dodecane- $d_{26}$ ) will be discussed first. Then the effect of exchanging the sodium counterion for tetrapropylammonium on the CMC in cyclohexane- $d_{12}$ will be considered. 


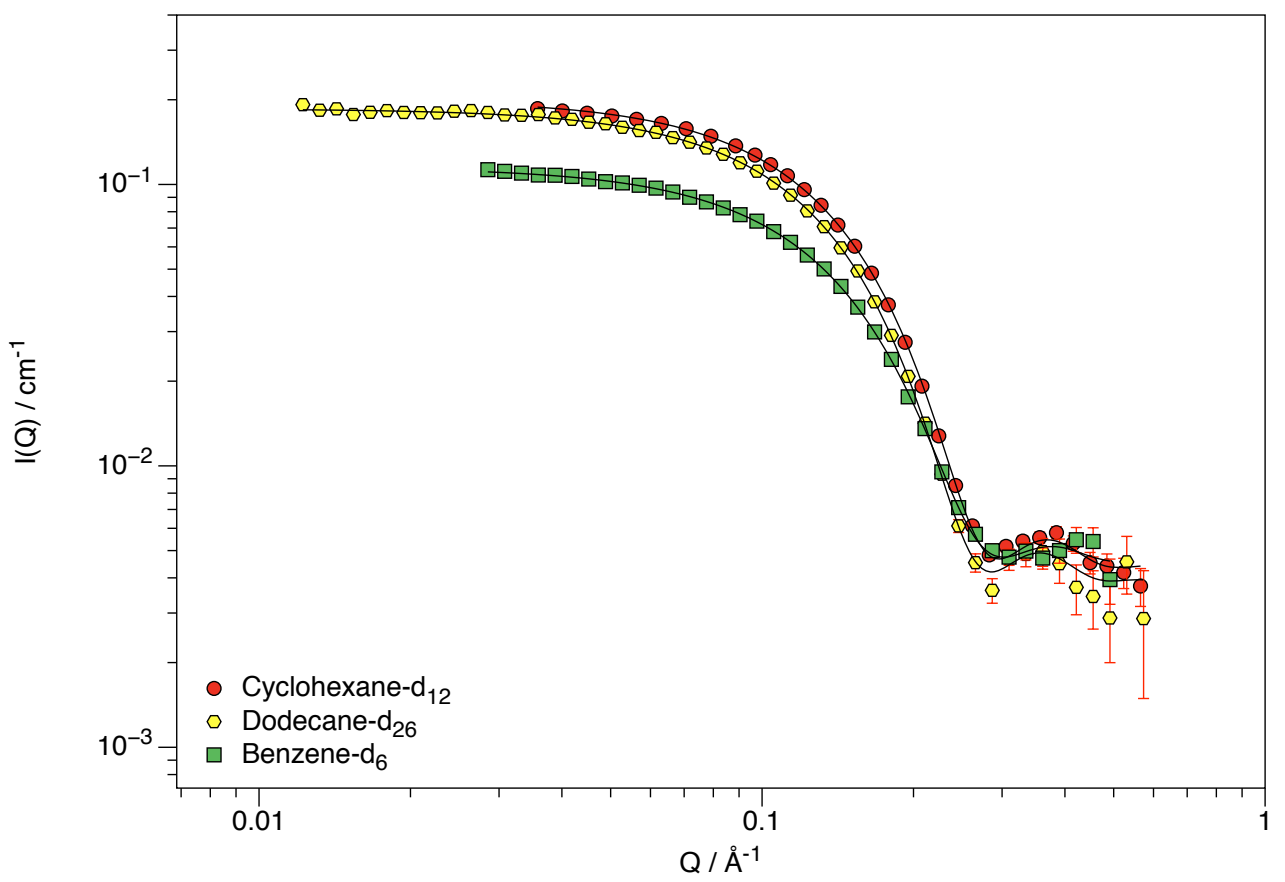

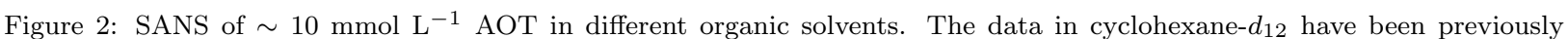
reported 22]. The aggregates can all be fit to a spherical form factor with a radius of $15-16 \AA$ with a Schulz dispersity $\sigma=0.08$, consistent with literature values for AOT in cyclohexane [22, 26].

\subsection{Solvent Variation}

The SANS curves for AOT in three different organic solvents at the highest concentrations studied $(\sim 10$ mmol L ${ }^{-1}$ ) are shown in Figure 2. The inverse micelles in all three solvents can be well described by a spherical form factor with a small Schulz dispersity $(\sigma=0.08)$. The fit radii are between 15 and $16 \AA$ consistent with previous SANS measurements of AOT inverse micelles in cyclohexane [22, 26]. Changing the organic solvent does not appear to influence the aggregates formed in the high concentration regime.

The serial dilution approach used to determine the CMC is the same as previously used for AOT in cyclohexane- $d_{12}$ [22, and the results are shown in Figure 3 . (The results in cyclohexane- $d_{12}$ are shown in Figure $3 \mathrm{a}$ for reference.) The concentration was decreased in logarithmic steps, and $I(Q)$ should decrease proportionally; indeed, this is the case. No detectable scattering could be measured at concentrations below the most dilute sample shown in Figure 3; scattering curves for these lower concentration solutions are shown in the Supplementary Data file.

By comparing the SANS curves in Figure 3 at different concentrations and in different solvents, it is apparent that the inverse micelles in all systems are similar. The SANS data can be fit using a spherical form factor with a small Schultz dispersity $(\sigma=0.08)$. The dispersity was fixed for fitting and plotting, and the radii were allowed to vary to obtain the best fits.

Using the best fit radii from the SANS data (shown in Figure 3), it is possible to determine the aggregation 


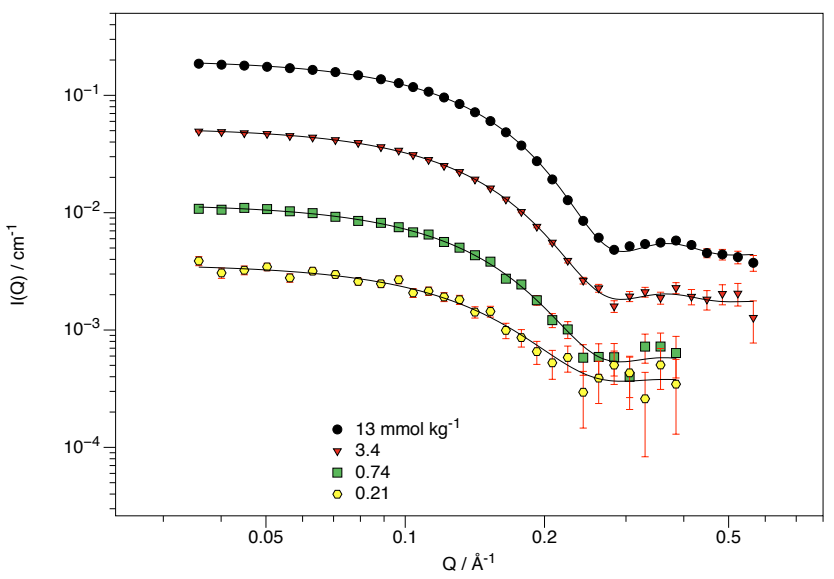

(a) AOT in cyclohexane- $d_{12} 22$

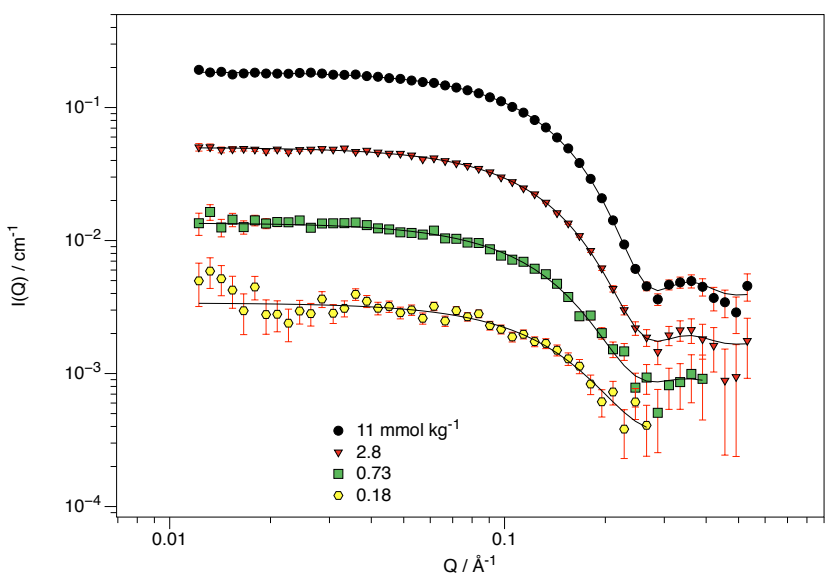

(b) AOT in dodecane- $d_{26}$

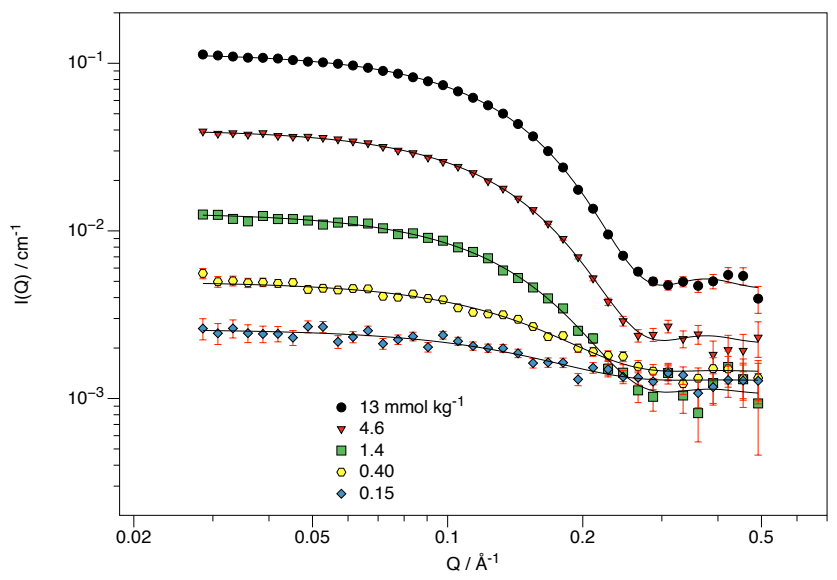

(c) AOT in benzene- $d_{6}$

Figure 3: SANS of AOT as a function of decreasing concentration in cyclohexane- $d_{12}$, dodecane- $d_{26}$, and benzene- $d_{6}$. Only measurements above the inverse micelle $\mathrm{CMC}$ are shown. The scattering curves for in the three solvents are similar, and all data were fit to a spherical form factor with a small Schulz dispersity $(\sigma=0.08)$. 


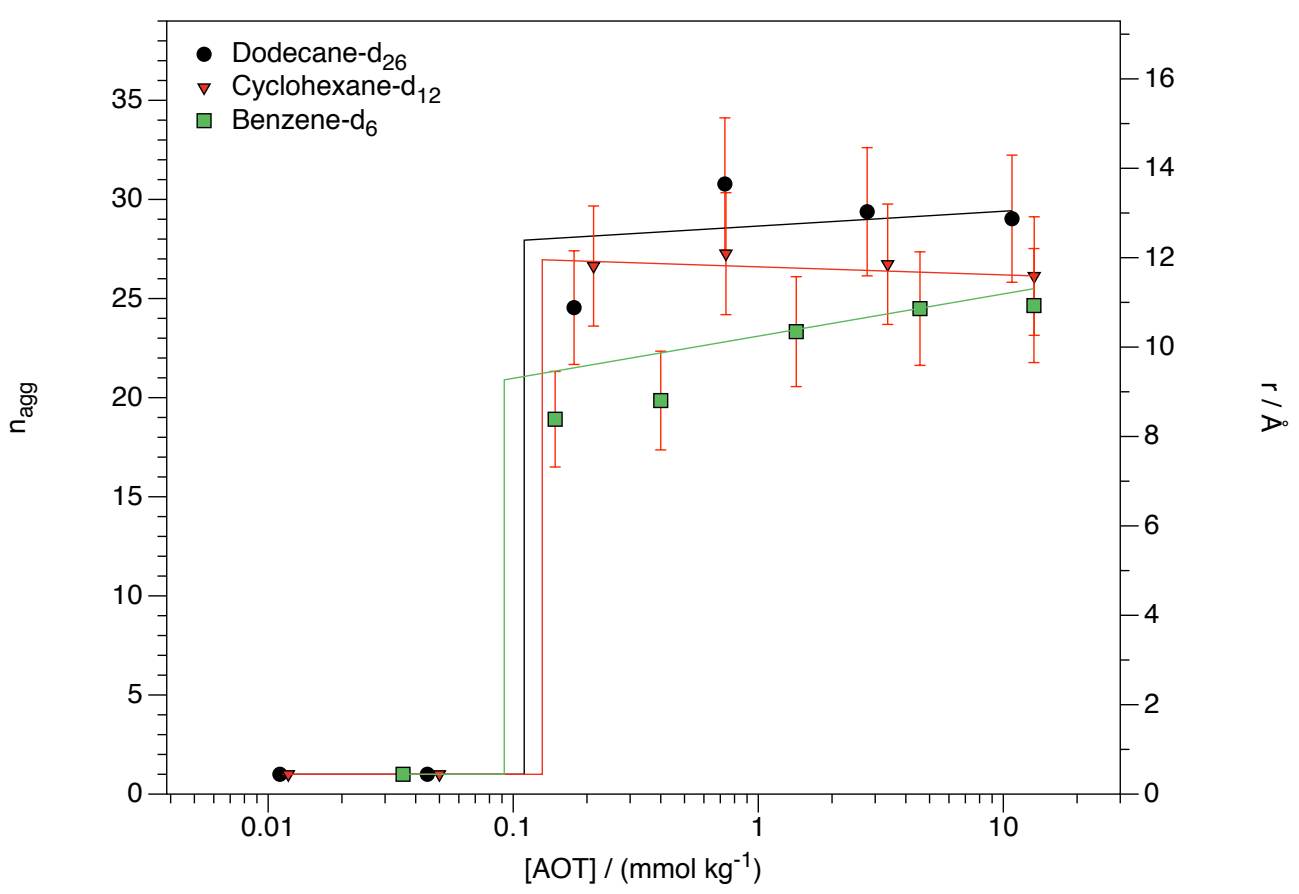

Figure 4: The inverse micelle CMC for AOT in different organic solvents in mmol kg-1. Both $n_{a g g}$ and the inverse micelle radius $r$ are shown as $n_{a g g}$ is a function solely of $r$ when the surfactant molecular volumes are equal. The CMCs are essentially identical, despite the solvents being chemically different.

number $\left(n_{\text {agg }}\right)$ of the surfactants at different concentrations. The value of $n_{\text {agg }}$ is determined from the volume of a spherical inverse micelle and the molecular volume $\left(v_{m}\right)$ of AOT, given by Equation 4 for spherical inverse micelles. This approach has previously been used for AOT aggregates studied by SANS [11].

$$
n_{\text {agg }}=\frac{4 \pi r^{3}}{3 v_{m}}
$$

This makes the assumption that no solvent mixes with the surfactant tails, which has been shown to be the case in extensive contrast-variation SANS studies of AOT water-in-oil microemulsions [40]. Some NMR studies of AOT at water-oil interfaces claim that oils penetrate into the surfactant tails [41, 42, but there is not sufficient evidence to apply this confidently and quantitatively to the determination of the aggregation number.

The values of $n_{\text {agg }}$ for AOT in the three solvents used in this study are shown in Figure 4 The CMC is defined as the midpoint between the lowest concentration where inverse micelles are detected and the highest concentration where they cannot be detected. Values of the CMC determined from Figure 4 are shown in Table 1

The aggregation number in dodecane is similar to that previously measured for AOT in cyclohexane using SANS 22, 26]. Between the highest AOT concentrations measured $\left(\sim 10 \mathrm{mmol} \mathrm{\textrm {kg } ^ { - 1 }}\right)$ and the CMC $\left(\sim 0.1 \mathrm{mmol} \mathrm{kg}^{-1}\right)$, the aggregation number is constant at roughly 28 and then drops to 1 below the CMC. 
Table 1: The inverse micelle CMC of AOT in different organic solvents

\begin{tabular}{lcc}
\hline Solvent & CMC (This study) $/\left(\mathrm{mmol} \mathrm{kg}^{-1}\right)$ & $\mathrm{CMC}$ (Literature) $)^{*} /\left(\mathrm{mmol} \mathrm{kg}^{-1}\right)$ \\
\hline Benzene & $0.09 \pm 0.06$ & 0.84 \\
Cyclohexane & $0.13 \pm 0.08$ & 0.25 \\
Dodecane & $0.11 \pm 0.07$ & - \\
Decane & - & 1.0 \\
\hline \multicolumn{2}{c}{${ }^{*}$ Benzene (SANS) [18, Cyclohexane (SANS) [26, Decane (SAXS) [25] }
\end{tabular}

The stepwise transition in $n_{\text {agg }}$ from inverse micelles to monomers is also observed for AOT in benzene; however, the value of $n_{a g g}$ is not constant over the whole concentration range. At high AOT concentrations, near $10 \mathrm{mmol} \mathrm{kg} \mathrm{kg}^{-1}, n_{\text {agg }}$ is similar to the values in cyclohexane and dodecane. At AOT concentrations near the CMC, the value of $n_{\text {agg }}$ was found to decrease slightly. The magnitude of this increase is not large, but it is greater than the standard error expected from SANS data fitting $( \pm 1 \AA)$. In the mass-action description of the process of micellization, although aggregates with different aggregation numbers can exist, only aggregates of size $n_{a g g}$ are present in significant concentrations above the CMC 43. The fact that $n_{\text {agg }}$ changes with concentration seems to contradict this description. However, it does agree with previous literature studies of the aggregation of AOT in benzene; $R_{g}$ (proportional to $n_{a g g}$ ) was found to decrease at low surfactant concentrations near the CMC [18. This suggests that there are minor differences in the solvophobic effect as the nonpolar solvent is changed.

\subsection{Counterion Variation}

To study the influence of varying the counterion on aggregation in organic solvents, the inverse micelle CMC for tetrapropylammonium-AOT (TPA-AOT) was measured and compared to that for NaAOT. This was done to explore possible variations in solvophobicity when hard inorganic cations are swapped for softer organic cations, which are expected to be more solvophilic. To determine the microstructure of the inverse micellar aggregates in solution, two labeled analogues have been studied, the standard TPA-AOT as well as the deuterium-labeled TPA-AOT- $d_{34}$ in cyclohexane- $d_{12}$. The SANS curves of the two surfactants at 14 mmol $\mathrm{kg}^{-1}$ are shown in Figure 5. The form factors are qualitatively similar, indicating that the two are present in a single aggregated species rather than as separate ions.

The two data sets were fit to a core-shell sphere model, and a simultaneous, co-refined fit was performed to determine how TPA and AOT are distributed throughout the inverse micelles. The scattering length density of the TPA cations (assumed to be in the core) are the same for the two samples, but the scattering length density of the AOT anions (assumed to be in the shell) was different depending on whether AOT or AOT- $d_{34}$ was used. The total radii of the inverse micelles are found to be similar to NaAOT (12 $\AA$ for TPA-AOT compared to $15 \AA$ for NaAOT), but there is significant size dispersity in the core. The best fit 


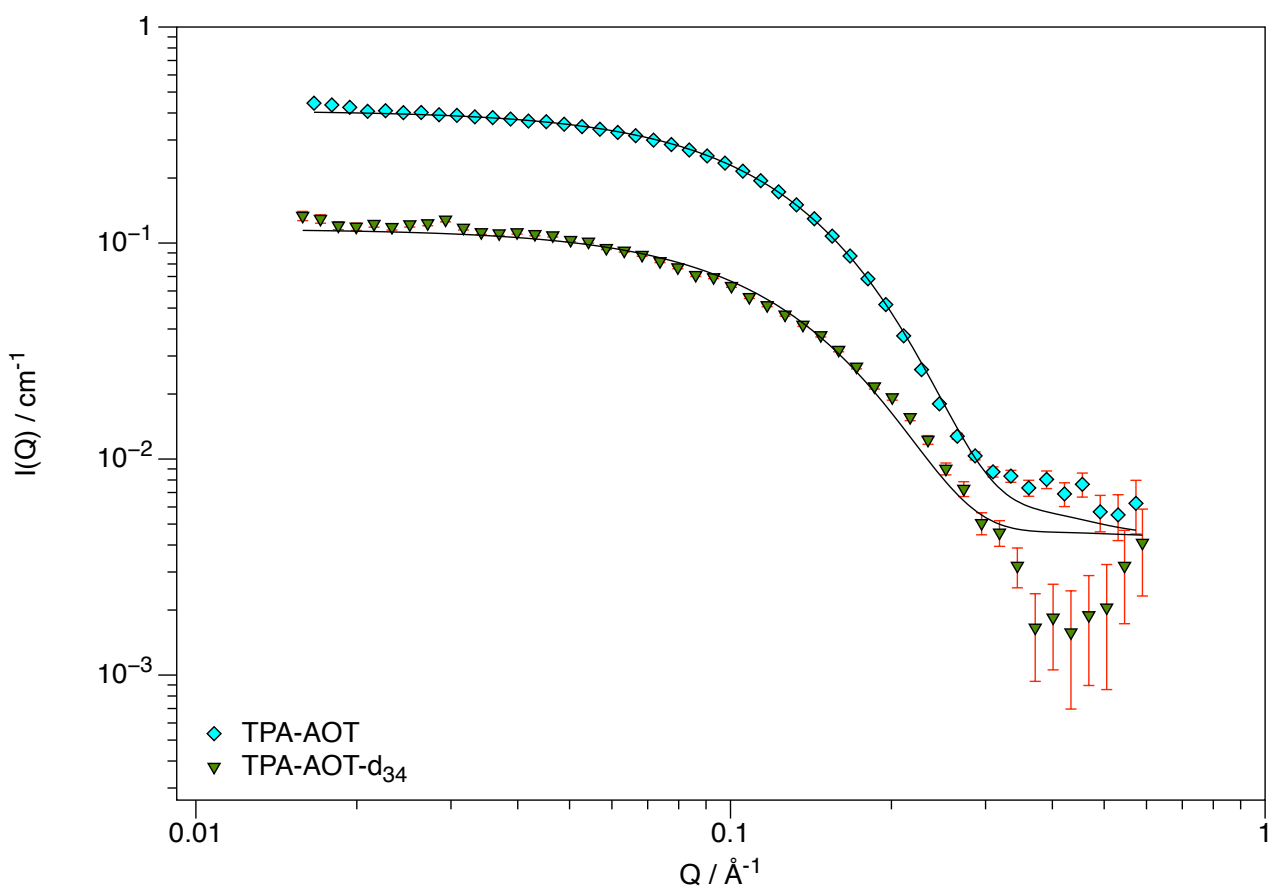

Figure 5: SANS of samples of TPA-AOT and TPA-AOT- $d_{34}$ at a concentration of $14 \mathrm{mmol} \mathrm{kg}^{-1}$ in cyclohexane- $d_{12}$. A simultaneous, co-refined fit was performed on the data to enable a more precise determination of the dimensions of the core and shell. The radius of the core, consisting of TPA, is found to be $2.3 \AA(\sigma=0.90)$, and the thickness of the shell, consisting of AOT, is found to be $10.2 \AA\left(\sigma_{t}=0.17\right)$.

core radius is $2.3 \AA(\sigma=0.90)$, and the best fit shell thickness is $10 \AA\left(\sigma_{t}=0.17\right)$. The DFT-calculated molecular volume of TPA is $282 \AA^{3}$, and the spherical volume of the inverse micelle cores is $50 . \AA^{3}$. This suggests that there is significant penetration of TPA into the shell.

AOT and TPA interpenetration is supported by the values of the scattering length density difference between the shell and solvent $\left(\Delta \rho_{s}\right)$, which deviates from the predicted experimental values assuming the shell is formed entirely of AOT anions. The fit value for $\Delta \rho_{s}$ for TPA-AOT is found to be $6.34 \times 10^{-6}$ $\AA^{-2}$ (predicted $\Delta \rho_{s}=6.09 \times 10^{-6} \AA^{-2}$ ) and for TPA-AOT- $d_{34}$ is found to be $3.16 \times 10^{-6} \AA^{-2}$ (predicted $\Delta \rho_{s}=0.85 \times 10^{-6} \AA^{-2}$ ). If this difference is assumed to originate solely from the penetration of TPA into the AOT shell, this would give a volume fraction of TPA of 0.21 for TPA-AOT and 0.36 for TPA-AOT- $d_{34}$.

SANS was measured for TPA-AOT in cyclohexane- $d_{12}$ as a function of decreasing concentration, shown in Figure 6 The sizes of the inverse micelles (core radius plus shell thickness) are found to be relatively independent of concentration, ranging from 11-13 A. The precision of the contrast between core and shell decreases as the concentration decreases due to the consequent decrease in the magnitude of $I(Q)$. However, as can be seen in Figure 6, scattering above the baseline is detectable; at even lower concentrations, no scattering could be detected (shown in the Supplementary Data file). This results in a value of the CMC of $0.19 \pm 0.12 \mathrm{mmol} \mathrm{kg}^{-1}$, essentially the same as for NaAOT in cyclohexane- $d_{12}$ (Table 1), within the error of 


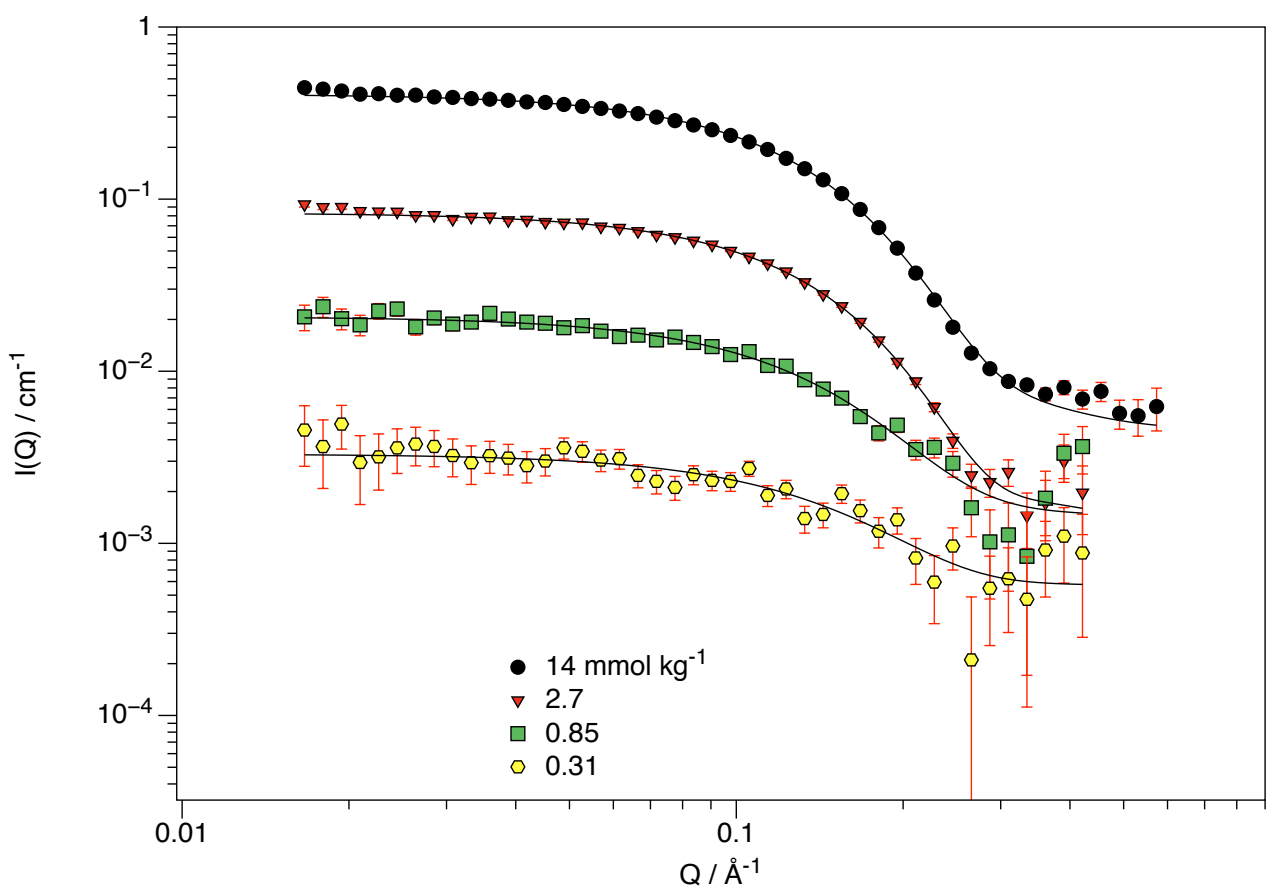

Figure 6: SANS of TPA-AOT in cyclohexane- $d_{12}$ as a function of surfactant concentration. Below these concentrations, no scattering above the solvent baseline can be detected, giving a value for the CMC of $0.19 \pm 0.12 \mathrm{mmol} \mathrm{kg}^{-1}$.

the measurements.

The values of $n_{\text {agg }}$ calculated using the $v_{m}$ calculated for the two surfactants and Equation 4 for NaAOT and TPA-AOT are shown in Figure 7. The values of $n_{\text {agg }}$ for TPA-AOT are lower than for NaAOT, and this suggests a difference in how the two surfactants aggregate. TPA-AOT is a bulkier species given the size of the soft TPA cation compared to the hard $\mathrm{Na}^{+}$cation. The same inverse micelle volume can be filled by a smaller number of surfactant molecules. Additionally, the fits to the SANS data in Figure 5 are consistent with TPA cation penetration into the AOT shell, and this enables stabilization of the polar core with a smaller number of surfactant monomers.

Despite the differences in the structure and $n_{\text {agg }}$ of NaAOT and TPA-AOT inverse micelles, the CMC for forming inverse micelles is similar, indicating that changing the counterion is a second order effect regarding influences on the CMC.

\section{Conclusions}

The effect of varying the solvophobicity of surfactants can now be considered in more detail. New highresolution SANS measurements have shown that there are no notable differences between the values of the CMC of sodium AOT in benzene, cyclohexane, and dodecane or TPA-AOT in cyclohexane, at least within the resolution of these measurements. Some differences in aggregate structure, either for low concentration 


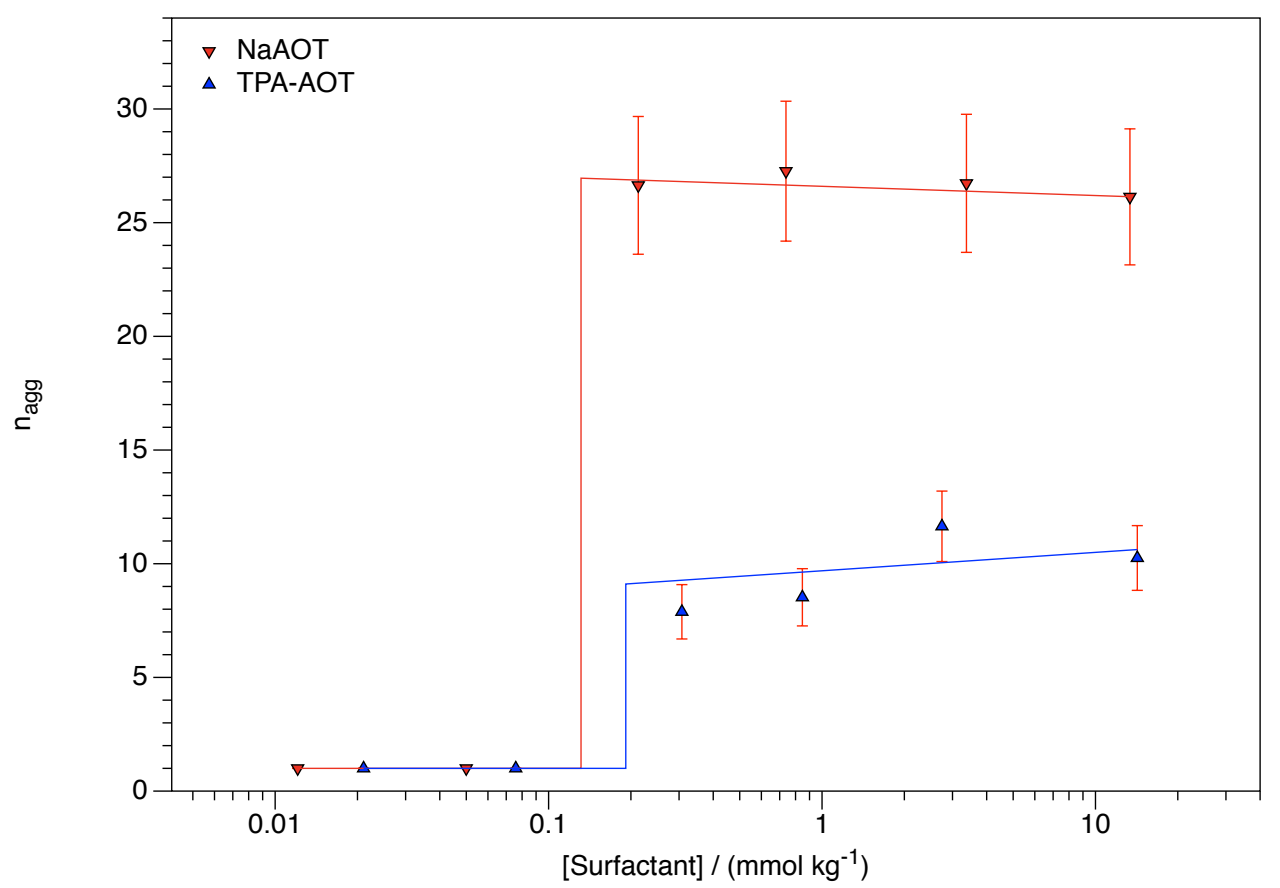

Figure 7: Inverse micelle CMCs for NaAOT and TPA-AOT in cyclohexane- $d_{12}$ in $\mathrm{mmol} \mathrm{kg}^{-1}$. Due to the large TPA cation (compared to $\mathrm{Na}^{+}$), the aggregation number of TPA-AOT is lower than for NaAOT. However, the value of the inverse micelle CMC is similar for the two surfactants.

AOT aggregates in benzene or TPA-AOT aggregates in cyclohexane, are apparent, but the onset of inverse micelle formation appears to be independent of the molecular variations studied here. The magnitude of the solvophobicity is too great to be influenced by the variations introduced, either because the counterions are too hard or the solvent intermolecular interactions are too weak. It is particularly surprising that there is no difference in the inverse micelle CMC between NaAOT and TPA-AOT within the resolution of these measurements, given that the counterions are extremes of hard and soft. Similar observations were recently made for the aggregation of anionic surfactants with tetralkylammonium counterions in water; $\mathrm{CH}_{2}$ groups in the surfactant cations and anions did not have an equivalent effect on the CMC [27. This appears to be broadly the case in nonaqueous solvents as well.

Using more variations than presented in this study will enable further development of relationships between the surfactant structure and inverse micelle CMC. One such possibility was shown in Figure 1 nonionic surfactants in an alkane. Additionally, the saturation concentration of both nonionic surfactants in alkanes and of perfluorinated alcohols in MEK depends on the number of solvophobic groups (ethylene oxide or $\mathrm{CF}_{2}$ units) [44, 45]. However, the literature available on these types of measurements is limited.

This paper does not demonstrate any significant effect of varying solvent-surfactant interactions for ionic surfactants in alkanes, but these materials are very interesting to study, given their applications as microemulsion stabilizers [8], as nanoreactors [9], and as charge control agents [10]. This provides a strong 
motivation to gain further understanding into their aggregation. These species, however, are highly solvophobic in nonpolar solvents, and the results in this study show that reasonable variations to the solvent and counterion that should influence the CMC do not appear to do so. This raises the question of how it would be possible to vary the CMC of ionic surfactants in nonpolar solvents. Making counterions harder or softer, for example, does not appear to be effective. New surfactant chemistry approaches will be needed to explore this further, and this will be very beneficial to develop a deeper understanding of aggregation in organic solvents.

\section{Acknowledgements}

GNS acknowledges Merck Chemicals Ltd. UK, an affiliate of Merck KGaA, Darmstadt, Germany, and the UK Engineering and Physical Sciences Research Council (EPSRC) for the provision of a CASE PhD studentship. The authors thank the UK Science and Technology Facilities Council (STFC) for allocation of beamtime at ISIS and grants toward consumables and travel. This work benefitted from SasView software, originally developed by the DANSE project under NSF award DMR-0520547.

\section{References}

[1] H. B. Klevens, Critical micelle concentrations as determined by refraction, J. Phys. Colloid Chem. 52 (1) (1948) $130-148$. doi:10.1021/j150457a013.

[2] H. B. Klevens, Structure and aggregation in dilate solution of surface active agents, J. Am. Oil Chem. Soc. 30 (2) (1953) 74-80. doi:10.1007/BF02635002.

[3] C. Tanford, Theory of micelle formation in aqueous solutions, J. Phys. Chem. 78 (24) (1974) 2469-2479. doi:10.1021/ j100617a012

[4] C. Tanford, The Hydrophobic Effect: Formation of Micelles and Biological Membranes, 2nd Edition, John Wiley \& Sons, Chichester, 1980.

[5] E. F. Williams, N. T. Woodberry, J. K. Dixon, Purification and surface tension properties of alkyl sodium sulfosuccinates, J. Colloid Sci. 12 (5) (1957) 452-459. doi:10.1016/0095-8522(57)90048-X

[6] H. C. Evans, 117. Alkyl sulphates. Part I. Critical micelle concentrations of the sodium salts, J. Chem. Soc. (1956) $579-586$ doi:10.1039/JR9560000579

[7] J. C. Ravey, M. Buzier, C. Picot, Micellar structures of nonionic surfactants in apolar media, J. Colloid Interface Sci. 97 (1) (1984) 9-25. doi:10.1016/0021-9797(84)90269-8

[8] J. Eastoe, B. H. Robinson, D. C. Steytler, D. Thorn-Leeson, Structural studies of microemulsions stabilised by aerosol-OT, Adv. Colloid Interface Sci. 36 (1991) 1-31. doi:10.1016/0001-8686(91)80027-H

[9] J. Eastoe, M. J. Hollamby, L. Hudson, Recent advances in nanoparticle synthesis with reversed micelles, Adv. Colloid Interface Sci. 128-130 (2006) 5-15. doi:10.1016/j.cis.2006.11.009

[10] G. N. Smith, J. Eastoe, Controlling colloid charge in nonpolar liquids with surfactants, Phys. Chem. Chem. Phys. 15 (2) (2013) 424-439. doi:10.1039/c2cp42625k

[11] M. J. Hollamby, R. Tabor, K. J. Mutch, K. Trickett, J. Eastoe, R. K. Heenan, I. Grillo, Effect of solvent quality on aggregate structures of common surfactants, Langmuir 24 (21) (2008) 12235-12240. doi:10.1021/1a8020854

[12] E. Ruckenstein, R. Nagarajan, Aggregation of amphiphiles in nonaqueous media, J. Phys. Chem. 84 (11) (1980) $1349-1358$. doi:10.1021/j100448a013.

[13] A. S. Kertes, Micellization, Solubilization, and Microemulsions. Volume 1, Plenum, London, 1977, Ch. Aggregation of surfactants in hydrocarbons. Incompatibility of the critical micelle concentration concept with experimental data., pp. $445-454$.

[14] A. Kitahara, T. Kobayashi, T. Tachibana, Light scattering study of solvent effect on micelle formation of Aerosol OT, J. Phys. Chem. 66 (2) (1962) 363-365. doi:10.1021/j100808a510. 
[15] S. Muto, K. Meguro, The determination of the CMC of surfactants in some organic solvents, Bull. Chem. Soc. Japan 46 (5) (1973) 1316-1320. doi:10.1246/bcs j.46.1316

[16] M. Ueno, H. Kishimoto, The behavior of Aerosol OT in organic solvents, Bull. Chem. Soc. Japan 50 (6) (1977) $1631-1632$. doi:10.1246/bcsj.50.1631

[17] Y.-C. Jean, H. J. Ache, Determination of critical micelle concentrations in micellar and reversed micellar systems by positron annihilation techniques, J. Am. Chem. Soc. 100 (3) (1978) 984-985. doi:10.1021/ja00471a061| URL http://pubs.acs.org/doi/abs/10.1021/ja00471a061

[18] N. Gorski, Y. M. Ostanevich, Critical micelle concentration (CMC) in the AOT-water-benzene system as determined by small-angle neutron scattering, Ber. Bunsenges. Phys. Chem. 95 (8) (1991) 871-875. doi:10.1002/bbpc.19910950805

[19] K. Mukherjee, S. P. Moulik, D. C. Mukherjee, Thermodynamics of micellization of Aerosol OT in polar and nonpolar solvents. A calorimetric study, Langmuir 9 (7) (1993) 1727-1730. doi:10.1021/la00031a020

[20] C.-C. Huang, K. L. Hohn, Tetrakis(dimethylamino)ethylene chemiluminescence (TDE CL) characterization of the CMC and the viscosity of reversed microemulsions, J. Phys. Chem. B 114 (8) (2010) 2685-2694. doi:10.1021/jp9077618

[21] S. M. Hashmi, A. Firoozabadi, Tuning size and electrostatics in non-polar colloidal asphaltene suspensions by polymeric adsorption, Soft Matter 7 (18) (2011) 8384-8391. doi:10.1039/C1SM05384A URL http://dx.doi.org/10.1039/C1SM05384A

[22] G. N. Smith, P. Brown, S. E. Rogers, J. Eastoe, Evidence for a critical micelle concentration of surfactants in hydrocarbon solvents, Langmuir 29 (10) (2013) 3252-3258. doi:10.1021/la400117s

[23] K. Kon-no, A. Kitahara, Kogyo Kagaku Zasshi 68 (1965) 2058.

[24] T. Kawai, K. Hamada, N. Shindo, K. Kon-no, Formation of AOT reversed micelles and W/O microemulsions, Bull. Chem. Soc. Japan 65 (10) (1992) 2715-2719. doi:10.1246/bcsj.65.2715

[25] T. Assih, F. Larché, P. Delord, Evolution of the radius of the inverse micelles at high dilution in the aerosol-OT/Water/ $n$ decane system, J. Colloid Interface Sci. 89 (1) (1982) 35-39. doi:10.1016/0021-9797(82)90117-5

[26] M. Kotlarchyk, J. S. Huang, S.-H. Chen, Structure of AOT reversed micelles determined by small-angle neutron scattering, J. Phys. Chem. 89 (20) (1985) 4382-4386. doi:10.1021/j100266a046

[27] P. Brown, C. Butts, R. Dyer, J. Eastoe, I. Grillo, F. Guittard, S. Rogers, R. Heenan, Anionic surfactants and surfactant ionic liquids with quaternary ammonium counterions, Langmuir 27 (8) (2011) 4563-4571. doi:10.1021/1a200387n

[28] G. N. Smith, S. Alexander, P. Brown, D. A. J. Gillespie, I. Grillo, R. K. Heenan, C. James, R. Kemp, S. E. Rogers, J. Eastoe, Interaction between surfactants and colloidal latexes in nonpolar solvents studied using contrast-variation small-angle neutron scattering, Langmuir 30 (12) (2014) 3422-3431. doi:10.1021/1a500331u

[29] Science \& Technology Facilities Council: ISIS. URL http://www.isis. Istfc.ac.uk/

[30] R. K. Heenan, S. E. Rogers, D. Turner, A. E. Terry, J. Treadgold, S. M. King, Small angle neutron scattering using Sans2d, Neutron News 22 (2) (2011) 19-21. doi:10.1080/10448632.2011.569531.

[31] I. Grillo, Small-angle neutron scattering and applications in soft condensed matter, in: R. Borsali, R. Pecora (Eds.), Soft Matter Characterization, Springer Netherlands, 2008, pp. 723-782. doi:10.1007/978-1-4020-4465-6\_13

[32] A. Guinier, G. Fournet, Small-Angle Scattering of X-Rays, John Wiley \& Sons, New York, 1955.

[33] D. J. Cebula, J. W. Goodwin, R. H. Ottewill, G. Jenkin, J. Tabony, Small angle and quasi-elastic neutron scattering studies on polymethylmethacrylate latices in nonpolar media, Colloid Polym. Sci. 261 (7) (1983) 555-564. doi:10.1007/ BF01526620.

[34] I. Marković, R. H. Ottewill, Small angle neutron scattering studies on nonaqueous dispersions of calcium carbonate Part 2. Determination of the form factor for concentric spheres, Colloid Polym. Sci. 264 (1) (1986) 65-76. doi:10.1007/BF01410309

[35] Sasview for small angle scattering analysis URL http://www.sasview.org/

[36] G. V. Schulz, Z. Phys. Chem. Abt. B 43 (1939) 25-46.

[37] M. Kotlarchyk, S.-H. Chen, Analysis of small angle neutron scattering spectra from polydisperse interacting colloids, J. Chem. Phys. 79 (5) (1983) 2461-2469. doi:10.1063/1.446055

[38] M. J. Frisch, G. W. Trucks, H. B. Schlegel, G. E. Scuseria, M. A. Robb, J. R. Cheeseman, G. Scalmani, V. Barone, B. Mennucci, G. A. Petersson, H. Nakatsuji, M. Caricato, X. Li, H. P. Hratchian, A. F. Izmaylov, J. Bloino, G. Zheng, J. L. Sonnenberg, M. Hada, M. Ehara, K. Toyota, R. Fukuda, J. Hasegawa, M. Ishida, T. Nakajima, Y. Honda, O. Kitao, H. Nakai, T. Vreven, J. A. Montgomery, Jr., J. E. Peralta, F. Ogliaro, M. Bearpark, J. J. Heyd, E. Brothers, K. N. Kudin, V. N. Staroverov, R. Kobayashi, J. Normand, K. Raghavachari, A. Rendell, J. C. Burant, S. S. Iyengar, J. Tomasi, M. Cossi, N. Rega, J. M. Millam, M. Klene, J. E. Knox, J. B. Cross, V. Bakken, C. Adamo, J. Jaramillo, R. Gomperts, R. E. Stratmann, O. Yazyev, A. J. Austin, R. Cammi, C. Pomelli, J. W. Ochterski, R. L. Martin, K. Morokuma, V. G. Zakrzewski, G. A. Voth, P. Salvador, J. J. Dannenberg, S. Dapprich, A. D. Daniels, Ö. Farkas, J. B. Foresman, J. V. Ortiz, J. Cioslowski, D. J. Fox, Gaussian 09 Revision D.01, Gaussian Inc. Wallingford CT 2009.

[39] A. F. Jalbout, Theoretical calculations of the molar volumes of atoms and molecules, J. Mol. Struct.: \{THEOCHEM\} 624 (1-3) (2003) 81-85. doi:10.1016/S0166-1280(02)00673-5

[40] J. Eastoe, K. J. Hetherington, D. Sharpe, J. Dong, R. K. Heenan, D. Steytler, Mixing of alkanes with surfactant monolayers in microemulsions, Langmuir 12 (16) (1996) 3876-3880. doi:10.1021/la960178s.

[41] Y. Hendrikx, H. Kellay, J. Meunier, Local properties of an AOT monolayer at the oil/water interfaces: NMR measurements, Europhys. Lett. 25 (9) (1994) 735-741. doi:10.1209/0295-5075/25/9/017

[42] C. A. Martin, L. J. Magid, Carbon-13 NMR investigations of Aerosol OT water-in-oil microemulsions, J. Phys. Chem. 85 (25) (1981) 3938-3944. doi:10.1021/j150625a046

[43] J. C. Berg, An Introduction to Interfaces \& Colloids: The Bridge to Nanoscience, World Scientific, Singapore, 2010. 
[44] K. Shinoda, M. Fukuda, A. Carlsson, Characteristic solution properties of mono-, di-, and triglyceryl alkyl ethers: lipophobicity of hydrophilic groups, Langmuir 6 (2) (1990) 334-337. doi:10.1021/1a00092a007

[45] L. J. Martin, Properties of fluorinated surfactants and polymers in organic solvents, Ph.D. thesis, University of Bristol (2005). 